\title{
Mutation-Aware Fault Prediction
}

\author{
David Bowes*, Tracy Hall ${ }^{\dagger}$, Mark Harman ${ }^{\ddagger},{\text { Yue } \mathrm{Jia}^{\ddagger} \text {, Federica Sarro }}^{\ddagger}$, and Fan $\mathrm{Wu}^{\ddagger}$ \\ *University of Hertfordshire, Hatfield, UK ${ }^{\dagger}$ Brunel University London, Uxbridge, UK \\ ${ }^{\ddagger}$ University College London, London, UK
}

\begin{abstract}
We introduce mutation-aware fault prediction, which leverages additional guidance from metrics constructed in terms of mutants and the test cases that cover and detect them. We report the results of 12 sets of experiments, applying 4 different predictive modelling techniques to 3 large realworld systems (both open and closed source). The results show that our proposal can significantly $(p \leq 0.05)$ improve fault prediction performance. Moreover, mutationbased metrics lie in the top $5 \%$ most frequently relied upon fault predictors in 10 of the 12 sets of experiments, and provide the majority of the top ten fault predictors in 9 of the 12 sets of experiments.
\end{abstract}

\section{CCS Concepts}

-Software and its engineering $\rightarrow$ Software testing and debugging;

\section{Keywords}

Software Fault Prediction, Software Defect Prediction, Mutation Testing, Software Metrics, Empirical Study

\section{INTRODUCTION}

Software fault prediction ${ }^{1}$ has been an active subject of research since the 1990s [12, 19, 22]. However, despite many proposals for different fault prediction techniques [4, 22, 66], no previous study has used mutation testing to improve the performance of predictive models.

In this paper we introduce mutation-aware fault prediction where several "mutation metrics" - derived by using mutation testing - are used as features to build a fault prediction model. We term such a model a 'mutation-aware' predictive model.

The key insight motivating the consideration of mutation awareness is that fault prediction performance must surely

${ }^{1}$ Please note that both the terms fault and defect have been used in the literature to refer to prediction models able to reveal faulty/defective software components. depend on the ability of the test suite to find faults. This is because the performance of a prediction is measured as its ability to predict the faults that will subsequently be revealed (by testing). Since mutation testing is generally used to assess the effectiveness of a test suite and previous work have demonstrated the coupling between real faults and mutation faults [3, 35], our motivation is to exploit this coupling: Mutation faults are coupled to real faults, so mutation faults should help predict real faults.

Consider the following scenario: The code has been tested by running a test suite, which may reveal some bugs. Unfortunately faults remain, we simply do not know where they reside; the dilemma faced by every tester. Our aim is to now predict where remaining faults are likely to reside. The majority of previous fault prediction studies has used static code metrics for this prediction task, yet there is dynamic information available resulting from the testing phase. The significant breakthrough of our study is that it is the first to use both static and dynamic test information. It is also the first to seed mutated faults which provides data allowing improved fault prediction.

In order to assess the effectiveness of mutation-aware fault prediction we define 40 mutation metrics (either 'static' or 'dynamic') and collect them using the popular (and publicly available) tool PITest [14]. Then, we empirically compare the performance of mutation-aware prediction models built using these metrics with respect to those of prediction models built using 39 traditional (mutation-unaware) static source code metrics that have been widely used in previous fault prediction work [46]. We also investigate whether the combined use of mutation metrics and source code metrics improves the accuracy of the resulting prediction model. Moreover, we analyse the extent to which different prediction techniques benefit from mutation awareness by using four different techniques (i.e., Logistic Regression, Random Forest, Naïve Bayes and J48), implemented in WEKA [21], to build both 'mutation-aware' and 'mutation-unaware' predictive models. Although we have our own tools for mutation testing and predictive modelling [26, 45, 50], we used publicly available third-party tools to avoid a source of potential experimenter bias, and to ensure that our results will have actionable findings for both mutation testing and predictive modelling.

We studied the impact on predictive performance of all 79 metrics on three large real-world systems. One of these systems is a closed source industrial system from a large telecom company (which we call TelCom). The other two, Eclipse (i.e., JDT Core) and Apache (i.e., DBUtils and NET com- 
bined), are open-source systems widely used in fault prediction studies. We use both closed and open-source systems to increase the potential for generalisability, though of course, as with all studies of software systems, caution is required when extrapolating. Taken together, these systems consist of over 220K Lines of Code, with a total of 263 real world faultive classes, revealed by over $11 \mathrm{~K}$ test cases.

To compare the performance of predictive models we use Matthews' Correlation Coefficient (MCC), which has recently been demonstrated to be one of the most reliable measures of predictive model performance [70]. For compatibility with other studies on fault prediction, we also include Precision and Recall, which are widely used metrics to assess the properties of predictive models.

The main contribution of this paper is the introduction of mutation-aware fault prediction, together with a study of the improvement mutation awareness conveys to fault prediction. The study involves 12 experimental evaluation scenarios, consisting of 4 machine learners (predictive modelling techniques) and 3 real world systems for which ground truth fault data has been carefully collected.

Our primary finding is that mutation awareness can significantly improve predictive performance and with large effect sizes. This is the first time any kind of mutation has been used to support fault prediction. Naturally, subsequent studies can further investigate/exploit this predictive improvement, perhaps in combination with other sets of metrics (e.g., process metrics). In this first study we present the empirical evidence that mutation metrics effect size on prediction outcomes can be large, thereby motivating and opening up this avenue of research.

In the following we briefly review the primary concepts underlying mutation testing and software fault prediction (Section 2). Then we present the research questions we aim to investigate (Section 3) and the design of the empirical study we carried out to answer them (Section 4). The results of the study and threats to its validity are discussed in Sections 5 and 6 , respectively. Section 7 summarises related work and Section 8 concludes.

\section{BACKGROUND}

\subsection{Mutation Testing and Mutation Metrics}

Mutation testing creates a variant of the program, called a mutant, into which a fault has been deliberately seeded [34]. Mutants are created by mutation operators, each of which seeds a particular class of faults, such as replacing one relational operator with another. A mutant is said to be 'killed' by a test case if the test case causes the original program and its mutant to behave differently [34, 77].

Originally, mutation testing was introduced in order to assess test effectiveness [18, 38], but it has also been used as a technique to guide the generation of test cases [20, 26, 62], and to improve test oracles [20,73]. One of the cornerstones of mutation testing is the hypothesis that at least some of the mutants will turn out to be coupled to real faults, such that test cases that are good at killing these mutants will also prove to be good at detecting real faults. There is empirical evidence to support this hypothesis [3,35].

This paper introduces a new application: using mutation testing to guide fault prediction. We insert mutants into a program and collect metrics about them, not to assess or generate test cases or oracles, but in the hope that the coupling between mutants and real faults will mean that at least some of these metrics will improve fault prediction.

We classify our mutation metrics as either 'static' or 'dynamic'. Static mutation metrics simply record the number of times a particular mutation operator can be applied, while dynamic mutation metrics record the number of times a particular class of mutant is killed by the test suite (the mutation score for this class of mutants), and/or whether the mutant is executed (covered) by the test suite. Dynamic mutation metrics thus take account of the test suite as well as the potential faults that can be seeded, while static mutation metrics merely take account of the kinds of faults that can be seeded. We distinguish between the two because, should it turn out that static metrics improve fault prediction, then this benefit can be exploited even when no testing information is available. More details on the metrics collected are reported in Section 4.4.

\subsection{Software Fault Prediction}

A predictive model for software fault-proneness generally exploits past data about software modules to classify the software modules as either predicted faulty or predicted nonfaulty. Predictive models infer a single aspect of the data (dependent variable) from some combination of other aspects of the data (independent variables). In the software fault prediction context, the dependent variable is represented by the faults contained in a software component while the independent variables may vary from project to project and can be related to different aspects of the software (e.g., source code metrics, process metrics).

The performance of the predictive model depends both on the modelling technique and the independent variables (i.e., metrics) used. Many predictive models have been investigated in the literature. Among them, machine learners and regression algorithms such as Decision Trees, Logistic Regression and Naïve Bayes are widely used [12, 36, 22]. Recently, also Search-Based approaches have been successfully exploited (e.g., [1, 11, 25, 45, 68]). However, according to recent systematic literature reviews $[22,66]$, the choice of a modelling technique seems to have less impact on the classification accuracy of a model than the choice of a metrics set. A selection of the most relevant variables (through feature subset selection) from the set of variables contained in the original dataset can be performed to eliminate variables that are irrelevant or of no predictive information value, thus enhancing learning efficiency and increasing predictive accuracy [22]. As the number of variables increases, finding an optimal feature subset might become intractable and different strategies might be needed (e.g., greedy or search-based algorithms). Feature selection can be carried out by searching the space of variable subsets, evaluating each one [17]. This is usually achieved by combining a machine learning algorithm - used to evaluate the usefulness of the feature set - with a search method (i.e., wrappers). The feature selection can be done also by ranking methods (i.e., filters) that evaluate the features according to heuristics based on general characteristics of the data. These methods are based on statistics, information theory, or on some function of the classifier's outputs.

The performance of a classification model is typically evaluated based on the confusion matrix. The matrix contains the following instances: True Positive (TP), faulty components correctly classified as faulty; False Negative (FN), 
faulty components incorrectly classified as non-faulty; False Positive (FP), non-faulty components incorrectly classified as faulty; True Negative (TN), non-faulty components correctly classified as non-faulty. The confusion matrix values are used to calculate a set of evaluation measures. Typical ones are Precision (measuring the proportion of the components classified as faulty which are actually faulty), Recall (measuring the proportion of faulty components classified as faulty), and F-Measure (which is the harmonic mean of precision and recall). However, it has been observed that these measures might be biased in the case of unbalanced data (i.e., minority and majority classes of very different sizes), which is often the case in fault prediction data. Thus, the use of balanced metrics, such as the Matthews Correlation Coefficient (MCC) has been recommended [70].

The MCC represents the correlation coefficient between the observed and predicted binary classifications and it is defined as follows:

$$
M C C=\frac{T P \times T N-F P \times F N}{\sqrt{(T P+F P)(T P+F N)(T N+F P)(T N+F N)}}
$$

MCC values range between -1 and +1 : +1 represents a perfect prediction, 0 no better than random prediction, and -1 indicates total disagreement between the prediction and the actual value. Contrary to other existing measures (e.g., Accuracy), MCC takes into account both true and false positives, and true and false negatives, so it is generally considered to be a balanced measure which can be used even if the data is unbalanced [70].

To validate predictive models a cross-validation process is typically used [4]. This process randomly splits the original dataset set into $k$-subsets (i.e., folds) of equal size (typically, $k=10)$. The model is trained by using the union of $k-1$ subsets (i.e., a training set) and validated on the remaining one that represents the test set. The process is repeated $k$ times, each time with a different testing set. In order to prevent overfitting an inner $k$-fold cross-validation can be also applied to the training set. To guarantee that the distribution of the faulty and non-faulty instances into training and test sets is representative of the distribution of the original dataset, stratification can be also used in the cross-validation process. Stratification allows us to mitigate cases of unbalanced data, such as using a fold with no faulty components, that might skew the result of the cross-validation process.

\section{RESEARCH QUESTIONS}

Our first research question investigates whether there is any effect on the performance of predictive models when they use mutation metrics. Unless the answer to this question is 'yes', then there is no point in further investigating mutation-aware predictive modelling.

RQ1: Is the performance of predictive modelling techniques improved when they have mutation metrics available?

In order to answer this research question we report on the difference in predictive model performance when the predictive model has all of the 79 metrics available (the "with mutation metrics' version), and when it only has available the 39 traditional metrics and no mutation metrics (the 'without mutation metrics' version). A 10-fold cross-validation is performed 50 times, each time with a different random shuffle of the data, in order to account for the stochastic nature of the overall predictive modelling algorithm. We use a two-tailed Wilcoxon non-parametric statistical test to check whether there are significant differences in the means for the 'with' and 'without' mutation metrics performance. We use the Wilcoxon test because we have no evidence to suggest that the performance of the predictive models is normally distributed over 50 runs. We use a two-tailed test, because we make no assumptions about which version of the predictive model will perform better in each case. We use the Matthews Correlation Coefficient (MCC), and Precision and Recall performance metrics to assess the predictive power of the fault prediction models.

If the answer to RQ1 is 'yes', then this motivates a more in-depth analysis of mutation metrics. However, an affirmative answer to this question does not provide sufficient scientific evidence, on its own, to motivate the use of mutationaware predictive modelling. It could be, for example, that mutation metrics only very occasionally improve the performance of a predictive modelling technique, or that they improve the performance more often, but only by a very slight amount. If the effect is too infrequent, or the size of effect too small, then there will still be no point in further study of mutation-aware predictive modelling. This motivates a second research question below:

RQ2: What is the effect size of improvements in predictive model performance due to mutation metrics?

We split this overall research question into three sub questions, which ask about the frequency and size of the effects of mutation testing on predictive modelling performance.

RQ2.1: What is the frequency with which each predictive modelling technique uses mutation metrics?

Using the same validation approach as for RQ1, we explore the results of the nested feature subset selection, for the four machine learners considered in this study. Therefore, for each subject and each learner, we obtain 500 different probabilistic model instances (50 repetitions of 10 -fold cross-validation), each of which has its own set of metrics, identified by feature subset selection. For each of the 79 metrics, we record which of the metrics occurs in these 500 probabilistic model instances. This frequency gives one indication of the importance of each metric, in terms of how often it is selected by feature subset selection, for each algorithm on each subject system. If some of the mutation metrics occur very frequently, then this strengthens the scientific evidence for the positive influence of mutation awareness on fault prediction.

RQ2.2: When a predictive modelling technique uses a mutation metric, what is the average predictive performance improvement due to this metric?

A particular metric may be frequently selected, yet with little effect on each occasion. Therefore, evidence for the frequent use of mutation metrics is encouraging, but insufficient on its own, to support strong scientific claims for mutation-aware predictive modelling. We therefore further investigate, by complementing the results of RQ2.1 with a study of the effect size for each of the mutation metrics: how much does it affect the predictive model performance on each occasion when it is used?

In order to measure this effect size, we remove the particular metric so that it is unavailable to the predictive model, and then measure the difference in predictive model performance with and without the availability of this metric. This gives us an effect size measurement for the usage of 
each metric when this is selected during the feature subset selection phase of each of the algorithm considered.

RQ2.3: For each mutation metric used on any occasion by a predictive modelling technique, what is the overall contribution of the metric to performance improvement?

Some metrics may have a big effect when they are used, but may, nevertheless, still be infrequent over all predictive model instances (i.e., such metrics are very useful, but only on a few occasions). If all the mutation metrics fall into this category, then that would be interesting, but would not constitute strong scientific evidence for mutation-aware fault protection. In order to capture the overall influence of each metric on the predictive models, we report on the product of the frequency and the effect size. This measures the average overall effect of a particular metric over all instances of a predictive modelling algorithm and subject system.

If we find that mutation metrics are selected frequently by feature subset selection, and that they can have a big effect when they are deployed into a predictive model, and that overall, their effect size is large, then we will have strong overall scientific evidence for the value and importance of mutation-aware fault prediction. We can then move on to study the individual mutation metrics (and classes of metrics) in more detail, which we do in RQ3 below.

RQ3: What is the relative performance of static and dynamic mutation metrics? In order to answer this question, we twice repeat the experimental procedure used for RQ1; once for static mutation metrics, and once for the dynamic mutation metrics. This allows us to investigate the individual specific advantage conveyed to predictive modelling using each of these sets of mutation metrics to complement the existing source code metrics.

Static mutation metrics can be computed in the absence of any test data, while dynamic mutation metrics also require a test suite. It is therefore interesting to see whether mutation metrics can benefit predictive modelling performance, even in the absence of a test suite; are static mutation metrics useful in their own right? We might expect that dynamic mutation metrics would convey greater advantage to a predictive modelling algorithm than static mutation metrics, because they also imbue the model with information about the test suite. If this is the case, then it suggests that other 'test suite aware metrics' might be investigated for their positive effects on fault prediction. On the other hand, it will be surprising and interesting if we find that such test suite information is not beneficial to fault prediction.

\section{EXPERIMENTAL SETUP}

In order to support replication ${ }^{2}$, this section provides details of the subject systems and their fault data, the source code and mutation metrics we used and the predictive modelling techniques with which we experimented.

\subsection{Subject Systems}

Table 1 provides details of the three subject systems used in this study. We can observe that there is variation in the size of the systems as well as the proportion of faulty classes

${ }^{2}$ The data used in this study (except for the closed source TelCom system which is covered by a Non-Disclose Agreement) is available at http://www0.cs.ucl.ac.uk/staff/F.Sarro/projects/

faultPrediction/index.html.

\begin{tabular}{l|r|r|r}
\hline & Apache & Eclipse & TelCom \\
\hline LOC Total & 22,430 & 113,106 & 84,731 \\
No. Classes & 74 & 727 & 1295 \\
No. Faulty Classes & 30 & 191 & 42 \\
\% Faulty Classes & $40.54 \%$ & $26.27 \%$ & $3.24 \%$ \\
No. Tests & 245 & 8,393 & 2,846 \\
\hline
\end{tabular}

Table 1: Contextual Information for the Subject Systems.

in each. The TelCom system has a very low proportion of faulty classes $(3.24 \%)$ making it a difficult subject for which to build good fault prediction models. Apache has the highest proportion of faulty classes at $40.54 \%$. Table 1 also shows that the number of tests used varies across the three systems. Apache has the lowest number of tests at 245 which may explain the relatively high faultiness in Apache.

\subsection{Fault Data}

We collected fault data from each of the three subject systems using the Śliwerski, Zimmerman and Zeller (SZZ) algorithm [71]. The SZZ algorithm matches the fault fix described in the bug tracking system with corresponding commits in the version control system that fixed that fault. This means that we were able to identify the fault insertion and fix points for every class in each of the subject systems and to label each class as either faulty or not-faulty for a given point in time. The data we collected has already been used in our previous work [23], where more details are provided.

\subsection{Source Code Metrics}

We collected 39 source code metrics (see Table 2) from each of the three subject systems. These metrics are wideranging and are frequently used in fault prediction studies [22]. We collected these metrics using the default settings of JHawk 1.8 [74], a tool previously identified as robust for collecting source code metrics from Java systems [46].

\subsection{Mutation Metrics}

As explained in Section 2.1 we classify our mutation metrics as either 'static' or 'dynamic'. In total five types of mutation metrics are collected as shown in Table 3. We used PITest [14] to perform mutation testing on each subject system. We chose this tool for mutation testing because we want to perform our analysis on large subjects and PITest is the most scalable mutation testing tool for Java programs. There are 7 mutation operators in PITest, which makes 8 mutation metrics of each type (with names ending ' 1 ' to ' 7 ' or 'A' for All). The numbered descriptions of the mutation operators are listed in Table 4. The number of mutants that can be generated using each of these operators yields a count. This count is a static mutation metric (i.e., MuNOM), because it is independent of any test suite. For each of the 8 static mutation metrics, we additionally compute 4 corresponding dynamic metrics. These count the number of times a mutant is covered (MuNOC), the number of times it is not covered (MuNNC), the mutation score for all mutants of this type (MuMS), and the score for only those which are covered (MuMSC). This gives us $32(4 \times 8)$ dynamic metrics, each of which draws into the predictive modelling framework information about the test suite; whether or not it can exercise a particular simulated fault (covered mutant), and whether it can detect this simulated fault (captured by mutation scores). 


\begin{tabular}{|r|l|l|}
\hline Index & Mutation Operator & Description \\
\hline 1 & Return Value Mutator & Mutates the return values of method calls. \\
\hline 2 & Negate Conditionals Mutator & Mutates conditionals between $==$ and $!=,<$ and $>=,>$ and $<=$. \\
\hline 3 & Increments Mutator & Replaces increments $(++)$ with decrements $(--)$ and vice versa. \\
\hline 4 & Void Method Calls Mutator & Removes void method calls. \\
\hline 5 & Conditionals Boundary Mutator & Replaces the relational operators between $<$ and $<=,>$ and $>=$. \\
\hline 6 & Invert Negatives Mutator & Inverts negation of integer and floating point numbers. \\
\hline 7 & Math Mutator & Swaps operations within groups: $\{+,-\},\{*, /, \%\},\{\&, 1, \uparrow\},\{<<,>>,>>>\}$. \\
\hline A & All of the above & Used to denote mutation metrics that count all the above metric instances. \\
\hline
\end{tabular}

Table 4: Mutation Operators

\begin{tabular}{|c|c|c|}
\hline Coding & Meaning & Origin \\
\hline AVCC & Average Cyclomatic Complexity & [47] \\
\hline $\mathrm{CBO}$ & Coupling Between Object Classes & [13] \\
\hline CCML & Cumulative Number of Comment Lines & [74] \\
\hline $\mathrm{CCOM}$ & Cumulative Number of Comments & {$[74]$} \\
\hline $\mathrm{COH}$ & Cohesion & [13] \\
\hline DIT & Depth of Inheritance Tree & [13] \\
\hline EXT & External Method Calls & [74] \\
\hline F-IN & Fan In & {$[29]$} \\
\hline FOUT & Fan Out & [29] \\
\hline HBUG & Halstead Cumulative Bugs & [24] \\
\hline HEFF & Halstead Effort & [24] \\
\hline HIER & Hierarchy Method Calls & [15] \\
\hline HLTH & Halstead Cumulative Length & [24] \\
\hline HVOL & Halstead Cumulative Volume & [24] \\
\hline INST & Instance Variables & [74] \\
\hline INTR & Number of Interfaces & [74] \\
\hline LCOM & Lack of Cohesion of Methods & [13] \\
\hline LCOM2 & Lack of Cohesion of Methods version 2 & [13] \\
\hline LMC & Local Method Calls & [74] \\
\hline MAXCC & Max Complexity & {$[74]$} \\
\hline MI & Maintainability Index & [61] \\
\hline MINC & Maintainability Index (No Comments) & [61] \\
\hline MOD & Number of Modifiers & [47] \\
\hline MPC & Message Passing Coupling & {$[42]$} \\
\hline $\mathrm{NCO}$ & Number of Commands & [74] \\
\hline NLOC & Lines Of Code & \\
\hline NOM & Number of Methods & [74] \\
\hline NOS & Number of Statements & [74] \\
\hline NQU & Number of Queries & [74] \\
\hline NSUB & Number of Subclasses & [13] \\
\hline NSUP & Number of Superclasses & [74] \\
\hline PACK & Number of Imported Packages & {$[74]$} \\
\hline $\mathrm{R}-\mathrm{R}$ & Reuse Ratio & [69] \\
\hline $\mathrm{RFC}$ & Response For class & [13] \\
\hline S-R & Specialization ratio & [69] \\
\hline SIX & Specialization index & [44] \\
\hline Superclass & SuperClass Name & [74] \\
\hline TCC & Total Complexity & [47] \\
\hline UWCS & UnWeighted Class Size & {$[74]$} \\
\hline
\end{tabular}

(www.virtualmachinery.com/jhawkmetricslist.htm).

\subsection{Machine Learning Techniques}

In this study we used four different classifiers (i.e., Naïve Bayes, Logistic Regression, J48, Random Forest) which cover a range of different techniques. Naïve Bayes (NB) [76] is a statistical technique which uses the combined probabilities of the different attributes to predict faultiness. Logistic Regression (LR) [16] is a regression technique which identifies the best set of weights for each attribute to predict the binary class. J48 is a Java implementation of the C4.5 [65] decision tree algorithm which uses entropy information to determine which attribute to use as decision nodes. Random Forest (RF) [9] is an ensemble technique which aggregates the predictions made by a collection of decision trees (each with a subset of the original set of attributes).

For each of these techniques we used the WEKA Wrapper Subset Selection Filter [40, 21] which performs a best first

\begin{tabular}{|l|l|l|}
\hline Metric & Description & Type \\
\hline MuNOM $^{S}$ & Number of generated mutants & Static \\
\hline MuNOC & Number of mutants covered by tests & Dynamic \\
\hline MuNNC & Number of mutants not covered by tests & Dynamic \\
\hline MuMS & Mutation score of generated mutants & Dynamic \\
\hline MuMSC & Mutation score of covered mutants & Dynamic \\
\hline
\end{tabular}

Table 3: Mutation metrics considered in our study. Key to colours/shading: Metrics in blue (dark grey for those reading a b/w copy) are static mutation metrics, while those in boldface are dynamic mutation metrics.

search algorithm to identify the subset of attributes that generalise best on the training set.

In order to validate the approaches we performed a 10fold cross validation with an inner 5 -fold cross-validation on the training set, and evaluated the results using MCC (see Section 2).

\section{RESULTS}

Our base results using only static code metrics are comparable if not better than results from other studies. Previous fault prediction work on Eclipse data, using static code metrics reported an average MCC of 0.412 with a standard deviation of 0.150 [22]. Our Eclipse results with static code metrics have an average MCC of 0.447 indicating that any improvement using mutation metrics improves even further than the majority of previous studies.

RQ1: Do Mutation Metrics Improve Performance?

Figure 1 contains three rows, each of which corresponds to one of the three subjects systems. Within each row, each of the four sets of box plots corresponds to one of the four different learning algorithms. Within each set there are box plots, labelled C, S, D, and A, which correspond to the MCC values obtained by the predictive models built using source code metrics alone, source code metrics augmented by static mutation metrics, source code metrics augmented by dynamic mutation metrics, all metrics (source code metrics augmented by static and dynamic mutation metrics)

In order to answer RQ1, consider the box plots labelled $\mathrm{C}$ and A within each row (we will return to the box plots $\mathrm{S}$ and $\mathrm{D}$ in our answer to RQ3). These give the overall performance (in terms of MCC) of the predictive modelling algorithms using the source code metrics alone compared to the performance using source code and mutation metrics. As these box plots reveal, for the TelCom and Apache systems, there is an improvement in performance for three of the four learners using mutation-aware predictive modelling. The effect is particularly pronounced for the Apache system, where performance is dramatically improved. As for Eclipse, there appears to be very little difference in the performances 


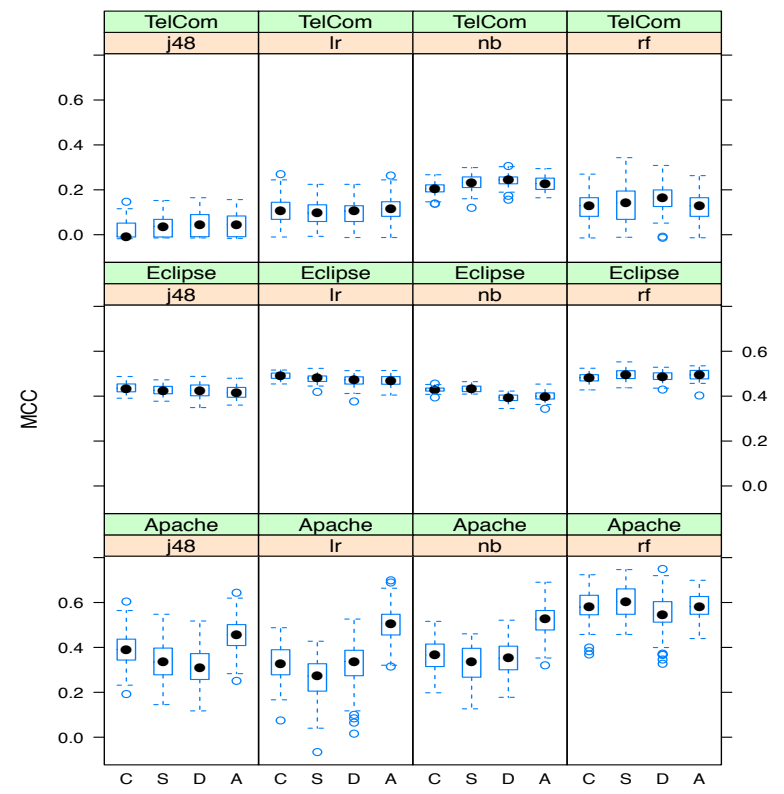

Figure 1: RQ1 and RQ3: MCC values for different predictive modelling algorithms on each of the three subjects. Key: $n b=$ Naïve Bayes, $l r=$ Logistic Regression, $r f=$ Random Forest, $j 48=\mathrm{J} 48 ; \mathrm{C}$ : source code metrics alone, S: source code metrics augmented by static mutation metrics, D: source code metrics augmented by dynamic mutation metrics, A: all metrics (source code metrics augmented by static and dynamic mutation metrics).

of any of the four algorithms, whether with or without mutation awareness. Furthermore, for Eclipse, all approaches to predictive modelling appear to show very little variance.

To further analyse these results, we performed an inferential statistical analysis of the differences between the median MCC values observed for each technique, the results of which are presented in Table 5a. These results confirm that fault prediction for the Apache system significantly benefits from mutation awareness. The industrial TelCom system also benefits from it, however the improvement is less pronounced. For the Eclipse system, there is a small reduction in performance, using mutation aware metrics. After performing the Benjamini-Hochberg correction for multiple statistical testing [5], only $p$-values $<0.001$ remain significant.

Tables $5 \mathrm{~b}$ and $5 \mathrm{c}$ provide a comparison of the differences (and their statistical significance) for the Precision and Recall values obtained using source code metrics alone, and using source code metrics augmented by mutation metrics (both static and dynamic). The results are consistent with those for the MCC performance metric. The Precision and Recall values give a more detailed explanation of the dramatic improvement achieved in MCC for the Apache system. Figure 2 shows the box plots of Precision and Recall values for this system. We can observe that mutation awareness improves the Recall of the predictive models, while retaining their Precision. As a result, more faulty classes are correctly identified using mutation metrics, without introducing any extra false positives (false alarms). The improvement in Recall for different systems results in being able to correctly predict additional faults: Apache with Naïve Bayes +7 (50\%), Eclipse with Random Forest $+2(2 \%)$ and TelCom with Naïve Bayes +1 (9\%).

(a) MCC

\begin{tabular}{l|c|c|c}
\hline & Apache & Eclipse & TelCom \\
\hline \multirow{2}{*}{ Naïve Bayes } & $<0.001$ & $<0.001$ & 0.018 \\
& $(0.182)$ & $(-0.030)$ & $(0.022)$ \\
\hline \multirow{2}{*}{ Logistic Regression } & $<0.001$ & $<0.001$ & 0.338 \\
& $(0.164)$ & $(-0.020)$ & $(0.005)$ \\
\hline \multirow{2}{*}{ Random Forest } & 0.858 & 0.005 & 0.920 \\
& $(-0.008)$ & $(0.013)$ & $(0.000)$ \\
\hline \multirow{2}{*}{ J48 } & $<0.001$ & $<0.001$ & 0.007 \\
& $(0.070)$ & $(-0.019)$ & $(0.057)$ \\
\hline
\end{tabular}

(b) Precision

\begin{tabular}{l|l|l|l}
\hline & Apache & Eclipse & TelCom \\
\hline \multirow{2}{*}{ Naïve Bayes } & $\begin{array}{l}0.049 \\
(0.032)\end{array}$ & $\begin{array}{l}0.020 \\
(-0.009)\end{array}$ & $\begin{array}{l}0.195 \\
(0.009)\end{array}$ \\
\hline \multirow{2}{*}{ Logistic Regression } & $<0.001$ & $<0.001$ & 0.871 \\
& $(0.085)$ & $(-0.019)$ & $(0.000)$ \\
\hline \multirow{2}{*}{ Random Forest } & $<0.001$ & 0.009 & 0.768 \\
& $(-0.028)$ & $(0.012)$ & $(-0.013)$ \\
\hline \multirow{2}{*}{ J48 } & 0.008 & $<0.001$ & $<0.001$ \\
& $(0.026)$ & $(-0.025)$ & $(0.143)$ \\
\hline
\end{tabular}

(c) Recall

\begin{tabular}{l|c|c|c}
\hline & Apache & Eclipse & TelCom \\
\hline \multirow{2}{*}{ Naïve Bayes } & $<0.001$ & $<0.001$ & $<0.001$ \\
& $(0.267)$ & $(-0.039)$ & $(0.024)$ \\
\hline \multirow{2}{*}{ Logistic Regression } & $<0.001$ & $<0.001$ & $\begin{array}{l}0.033 \\
(0.000)\end{array}$ \\
& $(0.167)$ & $(-0.016)$ & $(0.000)$ \\
\hline \multirow{2}{*}{ Random Forest } & $<0.001$ & $\begin{array}{l}0.023 \\
(0.067)\end{array}$ & $\begin{array}{l}0.317 \\
(0.010)\end{array}$ \\
\hline \multirow{2}{*}{ J48 } & $<0.001$ & $\begin{array}{c}0.681 \\
(-0.008)\end{array}$ & $\begin{array}{c}<0.001 \\
(0.024)\end{array}$ \\
\hline
\end{tabular}

Table 5: Two-tailed Wilcoxon test results are $p$-values that show the probability that we would obtain the difference in medians we observe, if the mutation metrics were to have no significant effect on predictive performance measured using MCC (a), Precision (b), and Recall (c). The number in brackets is the difference between the median values; where it is positive, it indicates that mutation metrics can improve predictive performance.

In conclusion, we find evidence that mutation awareness can improve the performance of predictive modelling, and that improvement is not restricted to only one technique, but can improve multiple techniques. There is also evidence that, where it does provide an improvement, the effect is highly significant. We therefore find evidence that mutation awareness is beneficial to predictive modelling.

RQ2: What is the effect size of improvements in predictive model performance due to mutation metrics? Having found evidence that mutation awareness can benefit predictive modelling, we now turn to a more detailed analysis of the frequency and (local and global) effect sizes of this benefit.

RQ2.1: What is the frequency with which each predictive modelling technique uses mutation metrics? Table 6 shows the top 10 most frequently used metrics for each of the 12 experiments (4 predictive models applied to 3 


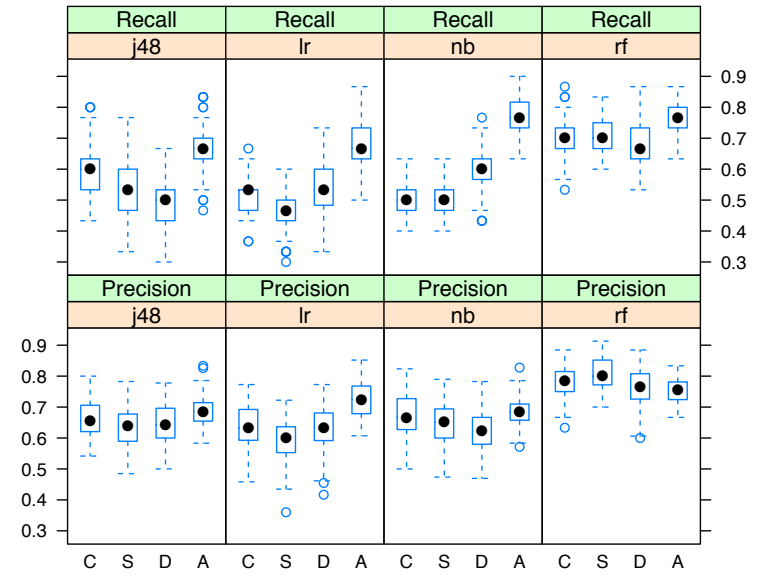

Figure 2: RQ1.Precision and Recall for Apache.

software systems). The number in brackets after each mutation name indicates the number of times (out of 500 folds in the cross fold validation) that the metric was used (for example, a number above 250 indicates that the corresponding metric is more likely to be used than not). We can observe that mutation metrics are very prevalent in every one of the 12 experiments, indicating that they are frequently useful in all of the predictive modelling scenarios considered. Table 7 gives a count of the number of mutation metrics that occur in the top 10 for each of the 12 experiments. As can be seen, the mutation metrics make up $50 \%$ or more of the top ten fault-predicting metrics for most cases and occur in the top 5\% (4/79) fault predictors in 10 of the 12 sets of experiments. Also, in every case (except 3: Random Forests (RF) applied to TelCom and J48 applied to Eclipse and TelCom) there is at least one mutation metric that is more likely to be used than not.

The results are also revealing for the traditional source code metrics: For example, our results provide evidence to suggest that different systems and different algorithms require different source code metrics for best performance. Clearly, the 'Fan Out' metric (FOUT) is highly applicable in many cases, being the most popular of all 79 metrics in 3 of the 12 experiments. Nevertheless, even this apparently useful metric, plays no role in the top 10 results for the J48 algorithm for any of the three systems studied. This finding, for traditional metrics, underscores the difficulty of fault prediction in general, and the impossibility of finding universally applicable techniques that always improve all fault predictions [72]. This observation tends to suggest that the scientific evidence for mutation awareness is all the more compelling, since it is so consistently beneficial to so many instances of the predictive modelling algorithms.

RQ2.2: When a predictive modelling technique uses a mutation metric, what is the average predictive performance improvement due to this metric? From RQ 2.1, we know that mutation metrics frequently provide benefit to all the predictive modelling algorithms and for all the programs studied. However, Table 7 simply considered the frequency with which a metric was used; this says nothing about the size of the effect, to which we now turn. Table 8 shows the effect size of the top ten (out of 79 ) met-

\begin{tabular}{|c|c|c|c|}
\hline & Apache & Eclipse & TelCom \\
\hline \multirow{10}{*}{ 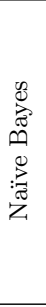 } & HEFF (492) & FOUT(500) & FOUT(465) \\
\hline & MuMS2(488) & $\operatorname{MINC}(500)$ & MuMS7(458) \\
\hline & MuMSA(340) & $\operatorname{NOM}(500)$ & MuMS4(427) \\
\hline & $\mathrm{R}-\mathrm{R}(306)$ & MuMSC4(471) & MuNOM $^{S} 7(418)$ \\
\hline & $\operatorname{MI}(266)$ & MuNOC2(342) & MuNNC3(416) \\
\hline & $\operatorname{LCOM}(155)$ & IuMSC3(309) & MuMSA(398) \\
\hline & MuNOM $^{S} \mathbf{3}(120)$ & MuMS1(226) & MI(373) \\
\hline & SIX(117) & $\operatorname{MAXCC}(210)$ & MuNNC7(317) \\
\hline & MuMSC3(85) & C & $\operatorname{SIX}(268)$ \\
\hline & $\mathrm{MuI}$ & 6) & MuMSC7(259) \\
\hline \multirow{10}{*}{ 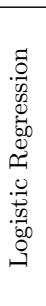 } & MuMSA(289) & $\operatorname{NOM}(500)$ & $\mathrm{CBO}(494)$ \\
\hline & SIX(285) & FOUT(418) & MuNOM $^{S} 7(443)$ \\
\hline & $\mathrm{Mu}$ & $\mathrm{Mu}$ & Mu \\
\hline & FOL & LCC & \\
\hline & MuNOC2(224) & $\operatorname{HEFF}(296)$ & MuNNC5(147) \\
\hline & MuN & 3) & MuNOM $^{S} 4(58)$ \\
\hline & $\mathrm{HEF}$ & & \\
\hline & MuN & 4) & $(45)$ \\
\hline & $\mathrm{R}-\mathrm{R}$ & & 43) \\
\hline & Mu & $\mathrm{TC}$ & FO \\
\hline \multirow{10}{*}{ 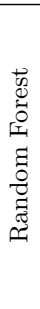 } & & & \\
\hline & $\mathrm{HE}$ & $\mathrm{F}($ & 1) \\
\hline & $7(285)$ & & \\
\hline & $\mathrm{CB}$ & & \\
\hline & F-IN $(266)$ & MuNOM $^{S} \mathbf{1}(295)$ & $\operatorname{MINC}(157)$ \\
\hline & MuNNC3(263) & $\operatorname{AVCC}(288)$ & MI(149) \\
\hline & $4(246)$ & $\mathrm{HFE}$ & $M_{1}$ \\
\hline & AVC & 8) & NL \\
\hline & $\operatorname{SIX}(212)$ & MuMSA(182) & $\operatorname{LCOM}(79)$ \\
\hline & MuNOM $^{S} 3(200)$ & HVOL(134) & $\operatorname{MAXCC}(75)$ \\
\hline \multirow{10}{*}{$\stackrel{\infty}{\leftrightarrows}$} & MuNOC2(265) & & \\
\hline & Mu & HLTH & $\mathrm{CB}$ \\
\hline & $\operatorname{AVCC}(138)$ & $\operatorname{AVCC}(151)$ & MuNOM $^{S} \mathbf{A}(202)$ \\
\hline & $\operatorname{MuNOM}^{S} \mathbf{A}(136)$ & FOUT(131) & $\operatorname{LCOM}(147)$ \\
\hline & MuNNC7(117) & MuMSCÁ( & MI(142) \\
\hline & MuNOM $^{S} \boldsymbol{7}(115)$ & Mu & SPIC, \\
\hline & NLOC(92) & $\mathrm{CBO}(106)$ & $\operatorname{AVCC}(129)$ \\
\hline & $\operatorname{HEFF}(89)$ & MuNOM $^{S} \mathbf{2}(105)$ & $\operatorname{NOS}(116)$ \\
\hline & TC3 & Mul & MuMSC1( \\
\hline & F-IN $(81)$ & $\operatorname{MuNOM}^{S} \mathbf{6}(96)$ & MuMSC4(105) \\
\hline
\end{tabular}

Table 6: RQ2.1. Top 10 most frequently used metrics for different datasets and classifiers.

rics in terms of their 'local' contribution to the performance of the algorithm. This effect size is 'local' in the sense that we measure the effect on the MCC performance assessment, only when the particular metric concerned is used.

Since MCC values lie between minus one and one, any improvement above 0.1 can be considered to be large and anything above 0.2 to be very large. For example, an improvement above 0.2 would be larger than the improvement that could be gained by any of the four algorithms on any of three systems studied, using the traditional source code metrics. As Table 8 reveals, when a mutation metric is used, its effect on the performance of the predictive model that uses it can be extremely large. In all but 1 of the 12 experiments, the effect size of at least one mutation metric is above 0.1 .

\begin{tabular}{lrrr}
\hline Technique & Apache & Eclipse & TelCom \\
\hline Naïve Bayes & 5 & 6 & 7 \\
Logistic Regression & 6 & 1 & 7 \\
Random Forest & 5 & 4 & 1 \\
J48 & 6 & 5 & 3 \\
\hline
\end{tabular}

Table 7: Frequency of mutation metrics in the top 10 metrics for different learners and datasets. 


\begin{tabular}{|c|c|c|c|}
\hline & Apache & Eclipse & TelCom \\
\hline \multirow{10}{*}{ 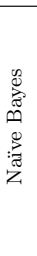 } & MINC $(0.507,5)$ & MuMSC7 $(0.138,7)$ & S-R $(0.394,1)$ \\
\hline & $\operatorname{HVOL}(0.437,4)$ & $\operatorname{HVOL}(0.127,4)$ & $\operatorname{NSUB}(0.349,1)$ \\
\hline & $\mathrm{CBO}(0.342,2)$ & $\operatorname{FOUT}(0.104,500)$ & $\operatorname{RFC}(0.155,4)$ \\
\hline & MuNOC2 $(0.317,4)$ & $\operatorname{TCC}(0.102,11)$ & MuMSC1 $(0.143,2)$ \\
\hline & $\operatorname{MuMSC7}(0.316,49)$ & MuNOM $^{S} \mathbf{5}(0.094,3)$ & $\operatorname{MuNOC} 4(0.126,45)$ \\
\hline & $\operatorname{MuNOM}^{S} \boldsymbol{7}(0.311,1)$ & MuMS2 $(0.086,28)$ & $\operatorname{MuNOM}^{S} \mathbf{5}(0.121,5)$ \\
\hline & MuNOC1 $(0.303,1)$ & $\operatorname{MAXCC}(0.082,210)$ & $\operatorname{Coh}(0.118,6)$ \\
\hline & $\operatorname{HEFF}(0.297,492)$ & MuMS4 $(0.078,11)$ & $\operatorname{HLTH}(0.111,152)$ \\
\hline & MuMSC1 $(0.292,21)$ & MuNOM $^{S} \mathbf{2}(0.074,20)$ & $\mathrm{NCO}(0.107,2)$ \\
\hline & MuNNC7 $(0.287,5)$ & $\operatorname{MINC}(0.074,500)$ & $\operatorname{NOM}(0.107,5)$ \\
\hline \multirow{10}{*}{ 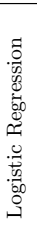 } & MuMSC7 $(0.482,16)$ & MuMSC2 $(0.124,29)$ & MuNNC2 $(0.384,2)$ \\
\hline & MuMSCA $(0.428,94)$ & $\operatorname{FOUT}(0.124,418)$ & $\operatorname{Coh}(0.379,1)$ \\
\hline & $\operatorname{MAXCC}(0.404,24)$ & MuMS4(0.123,21) & MuMSC2 $(0.371,1)$ \\
\hline & $\operatorname{NOM}(0.403,16)$ & F-IN $(0.121,29)$ & $\operatorname{HVOL}(0.352,1)$ \\
\hline & $\mathrm{CBO}(0.400,33)$ & MuMS2 $(0.120,27)$ & MuNOM $^{S} \mathbf{A}(0.316,2)$ \\
\hline & F-IN $(0.400,13)$ & $\operatorname{MuNOM}^{S} \mathbf{6}(0.113,15)$ & $\operatorname{MuNNC1}(0.287,7)$ \\
\hline & $\operatorname{HLTH}(0.399,12)$ & MuNNC6(0.110,7) & $\operatorname{MuMSC1}(0.281,6)$ \\
\hline & MuMSC2(0.393,12) & MuNNC2(0.100,46) & MuNOC5 $(0.275,4)$ \\
\hline & MuMSC1 $(0.382,17)$ & MuMSA(0.099,40) & $\operatorname{HLTH}(0.273,9)$ \\
\hline & $\operatorname{MuMSA}(0.380,289)$ & $\operatorname{AVCC}(0.097,83)$ & MuNOM $^{S} \mathbf{5}(0.272,8)$ \\
\hline \multirow{10}{*}{ 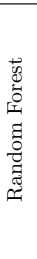 } & $\operatorname{LCOM}(1.067,2)$ & MuNOM $^{S} \boldsymbol{7}(0.687,1)$ & MuNNC6(1.006,8) \\
\hline & $\operatorname{MuNOM}^{S} \mathbf{2}(0.701,3)$ & $\operatorname{MI}(0.581,25)$ & $\operatorname{HVOL}(1.004,4)$ \\
\hline & $\operatorname{MuNOCA}(0.686,13)$ & $\operatorname{MINC}(0.576,5)$ & $\operatorname{MuNOM}^{S} \mathbf{2}(1.002,8)$ \\
\hline & MuNNC1 $(0.638,4)$ & MuNOM $^{S} \mathbf{A}(0.576,12)$ & $\operatorname{MuMSC7}(1.002,9)$ \\
\hline & $\operatorname{HLTH}(0.583,2)$ & $\operatorname{MuNOM}^{S} \mathbf{2}(0.572,11)$ & LCOM2 $(0.978,11)$ \\
\hline & $\operatorname{MI}(0.583,1)$ & $\operatorname{SIX}(0.564,7)$ & MuMS2 $(0.975,42)$ \\
\hline & FOUT $(0.543,10)$ & F-IN $(0.549,13)$ & $\operatorname{MuNOM}^{S} \mathbf{5}(0.971,17)$ \\
\hline & $\operatorname{MuNNCA}(0.540,26)$ & MuNOC3(0.547,12) & MuMS5 $(0.971,33)$ \\
\hline & $\operatorname{NLOC}(0.515,13)$ & MuNNC6(0.543,21) & MuNOC6 $(0.968,3)$ \\
\hline & $\mathrm{TCC}(0.514,11)$ & $\mathrm{CBO}(0.543,80)$ & MuMS7 $(0.961,14)$ \\
\hline \multirow{10}{*}{$\stackrel{\infty}{\stackrel{\infty}{\hookrightarrow}}$} & MuNOC2 $(0.518,265)$ & $\mathrm{NLOC}(0.238,54)$ & $\operatorname{HVOL}(0.449,17)$ \\
\hline & $\operatorname{NSUP}(0.481,14)$ & MuNOM $^{S} \mathbf{A}(0.218,32)$ & $\operatorname{TCC}(0.407,28)$ \\
\hline & $\operatorname{RFC}(0.479,21)$ & HLTH $(0.218,223)$ & MuNOC3 $(0.396,22)$ \\
\hline & MuMS2(0.462,227) & $\operatorname{MAXCC}(0.201,28)$ & MuNNC7 $(0.393,22)$ \\
\hline & MuMS5(0.451,27) & $\operatorname{AVCC}(0.194,151)$ & $\operatorname{MINC}(0.383,30)$ \\
\hline & MuNOM $^{S} \mathbf{1}(0.438,43)$ & $\operatorname{MuNOCA}(0.194,48)$ & $\operatorname{NSUB}(0.383,32)$ \\
\hline & MuNOM $^{S} \mathbf{3}(0.437,24)$ & FOUT $(0.193,131)$ & MuNNC1 $(0.372,63)$ \\
\hline & HBUG $(0.435,32)$ & MuMSC3 $(0.187,75)$ & $\operatorname{HBUG}(0.367,135)$ \\
\hline & $\mathrm{CBO}(0.435,45)$ & $\operatorname{MuNOC5}(0.185,32)$ & $\operatorname{CCOM}(0.363,53)$ \\
\hline & $\operatorname{MINC}(0.429,55)$ & MuMS3(0.183,60) & $\operatorname{MuNOM}^{S} \boldsymbol{7}(0.356,74)$ \\
\hline
\end{tabular}

Table 8: RQ2.2. Contribution of metric towards MCC(training) when using Naïve Bayes, Logistic Regression and J48.

These results suggest that where a mutation metric is used, its effect on the performance of a predictive model is large. However, these results cover only the local effect size, so we now study the global effect sizes in RQ2.3.

RQ2.3: For each mutation metric used on any occasion by a predictive modelling technique, what is the overall contribution of the metric to performance improvement? Table 9 shows the effect size of the top ten (out of 79) metrics in terms of their 'global' contribution to the performance of the algorithm. This effect size is 'global' in the sense that we measure the effect on the MCC performance assessment, across every use of the metric, counting its effect as 0.0 for all folds that fail to use it. As can be seen from the table, mutation metrics have a high global effect size in many cases. In 9 out of the 12 experiments, more than half of the top 10 global effect size metrics are mutation metrics. For the Apache system, there is a mutation metric that has a large effect size $(\geq 0.2)$ for all four algorithms studied. For the other two systems, the effect sizes are lower for the mutation metrics, but they are also lower for all metrics overall, indicating that, for these systems, there is seldom a single metric that can have a big impact on its own.

RQ3: What is the relative performance of static and dynamic mutation metrics?

Consider again, Figures 1 and 2, which we used to answer RQ1. Each figure also reports results for the separate effect on predictive model performance of static and dynamic

\begin{tabular}{|c|c|c|c|}
\hline & Apache & Eclipse & TelCom \\
\hline \multirow{10}{*}{ 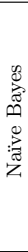 } & $\operatorname{HEFF}(0.292,492)$ & FOUT $(0.104,500)$ & FOUT $(0.094,465)$ \\
\hline & MuMS2(0.252,488) & $\operatorname{MINC}(0.074,500)$ & MuNNC3 $(0.056,416)$ \\
\hline & $\operatorname{MuMSA}(0.152,340)$ & MuMSC4 $(0.046,471)$ & MuMS7 $(0.055,458)$ \\
\hline & $\mathrm{MI}(0.073,266)$ & $\operatorname{MAXCC}(0.034,210)$ & $\operatorname{MuMSA}(0.055,398)$ \\
\hline & $\mathrm{R}-\mathrm{R}(0.063,306)$ & MuNOC2 $(0.029,342)$ & MuMS4 $(0.054,427)$ \\
\hline & $\operatorname{LCOM}(0.042,155)$ & $\operatorname{NOM}(0.025,500)$ & $\operatorname{SIX}(0.051,268)$ \\
\hline & MuNOM $^{S} \mathbf{3}(0.036,120)$ & MuMS1 $(0.023,226)$ & MuNOM $^{S} \boldsymbol{7}(0.049,418)$ \\
\hline & $\operatorname{MuMSCA}(0.033,66)$ & MuMSC3 $(0.021,309)$ & $\mathrm{MI}(0.047,373)$ \\
\hline & MuMSC7 $(0.031,49)$ & MuMS6 $(0.018,254)$ & MuNNC4 $(0.042,225)$ \\
\hline & MuMSC3 $(0.021,85)$ & MuMSC5 $(0.018,206)$ & $\mathrm{CBO}(0.036,238)$ \\
\hline \multirow{10}{*}{ 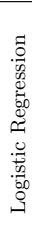 } & MuMSA $(0.220,289)$ & FOUT $(0.103,418)$ & MuNNC4 $(0.085,314)$ \\
\hline & $\operatorname{MuMS2}(0.212,284)$ & $\operatorname{NOM}(0.069,500)$ & $\mathrm{CBO}(0.078,494)$ \\
\hline & $\operatorname{SIX}(0.189,285)$ & MuMSC3 $(0.047,378)$ & MuNOM $^{S} \boldsymbol{7}(0.074,443)$ \\
\hline & MuNOC2 $(0.142,224)$ & $\operatorname{MAXCC}(0.046,263)$ & $\operatorname{SIX}(0.057,277)$ \\
\hline & $\operatorname{HEFF}(0.132,195)$ & $\operatorname{HEFF}(0.045,296)$ & MuNOC6 $(0.050,292)$ \\
\hline & FOUT $(0.128,233)$ & $\mathrm{TCC}(0.038,237)$ & MuNNC5 $(0.022,147)$ \\
\hline & MuNOM $^{S} \mathbf{3}(0.118,204)$ & $\operatorname{LCOM}(0.036,303)$ & FOUT $(0.020,41)$ \\
\hline & MuNOC1 $(0.092,172)$ & $\operatorname{NLOC}(0.035,254)$ & $\operatorname{CCML}(0.014,31)$ \\
\hline & MuMS4 $(0.086,128)$ & $\mathrm{R}-\mathrm{R}(0.033,257)$ & LCOM2 $(0.014,67)$ \\
\hline & $\operatorname{MuMSCA}(0.080,94)$ & $\operatorname{RFC}(0.028,248)$ & $\operatorname{CCOM}(0.013,64)$ \\
\hline \multirow{10}{*}{ 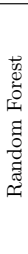 } & MuNOC2 $(0.400,463)$ & $\operatorname{NOM}(0.521,500)$ & $\operatorname{AVCC}(0.910,500)$ \\
\hline & $\operatorname{HEFF}(0.280,371)$ & $\operatorname{FOUT}(0.488,479)$ & $\mathrm{CBO}(0.554,321)$ \\
\hline & $\mathrm{F}-\mathrm{IN}(0.217,266)$ & HBUG $(0.335,311)$ & FOUT $(0.430,234)$ \\
\hline & MuNNC7 $(0.213,285)$ & MuNNC1 $(0.319,309)$ & $\operatorname{HLTH}(0.397,221)$ \\
\hline & MuNNC3(0.196,263) & MuNOM $^{S} \mathbf{1}(0.304,295)$ & $\operatorname{MINC}(0.283,157)$ \\
\hline & $\mathrm{CBO}(0.190,270)$ & $\operatorname{AVCC}(0.291,288)$ & $\operatorname{MI}(0.277,149)$ \\
\hline & MuNOM $^{S} \mathbf{4}(0.183,246)$ & MuNNC2 $(0.243,238)$ & $\operatorname{NOS}(0.238,133)$ \\
\hline & $\operatorname{AVCC}(0.168,222)$ & $\operatorname{HEFF}(0.243,238)$ & $\operatorname{MuNNCA}(0.204,110)$ \\
\hline & $\operatorname{SIX}(0.165,212)$ & MuMSA $(0.181,182)$ & $\mathrm{NCO}(0.180,97)$ \\
\hline & MuNOM $^{S} \boldsymbol{7}(0.144,196)$ & $\operatorname{HVOL}(0.140,134)$ & $\operatorname{NLOC}(0.144,80)$ \\
\hline \multirow{10}{*}{$\stackrel{\infty}{\stackrel{十}{\hookrightarrow}}$} & MuNOC2 $(0.274,265)$ & $\operatorname{NOM}(0.160,500)$ & $\operatorname{HEFF}(0.150,226)$ \\
\hline & MuMS2(0.210,227) & $\operatorname{HLTH}(0.097,223)$ & $\mathrm{CBO}(0.140,209)$ \\
\hline & $\operatorname{AVCC}(0.104,138)$ & $\operatorname{AVCC}(0.059,151)$ & MuNOM $^{S} \mathbf{A}(0.133,202$ \\
\hline & MuNOM $^{S} \mathbf{A}(0.102,136)$ & FOUT $(0.051,131)$ & HBUG $(0.099,135)$ \\
\hline & MuNOM $^{S} \boldsymbol{7}(0.090,115)$ & MuMSCA $(0.040,122)$ & $\operatorname{LCOM}(0.099,147)$ \\
\hline & MuNNC7 $(0.089,117)$ & $\mathrm{CBO}(0.036,106)$ & $\operatorname{MI}(0.095,142)$ \\
\hline & NLOC $(0.068,92)$ & MuMSC4 $(0.036,119)$ & $\operatorname{AVCC}(0.082,129)$ \\
\hline & MuNNC3 $(0.063,86)$ & MuNOM $^{S} \mathbf{2}(0.033,105)$ & $\operatorname{NOS}(0.080,116)$ \\
\hline & F-IN $(0.062,81)$ & MuMSC2 $(0.033,94)$ & $\operatorname{Coh}(0.069,105)$ \\
\hline & MuMS1 $(0.058,70)$ & MuMSC7 $(0.032,99)$ & MuMSC1 $(0.069,105)$ \\
\hline
\end{tabular}

Table 9: RQ2.3. Average Contribution of metric towards MCC(training) when using Naïve Bayes, Logistic Regression and $\mathrm{J} 48$.

mutation metrics. The box plots labelled 'S' give the MCC values (Figure 1) and precision and recall values (Figure 2) for predictive models that use source code metrics and static mutation metrics. The box plots labelled ' $D$ ' give MCC values and precision and recall values for predictive models that use source code metrics and dynamic mutation metrics. As can be seen from the figures, neither of the MCC values, nor the precision and recall values are noticeably improved for either S or D. Indeed, if anything, these box plots tend to suggest that either $\mathrm{S}$ or $\mathrm{D}$ on their own are unhelpful to predictive modelling performance. Nevertheless, when combined, the box plots labelled 'A' (All), indicate significant potential benefit as observed in answer to RQ1.

The results of the inferential statistical analysis of the performance improvement due to static (Table 10a) and dynamic (Table 10b) mutation metrics confirm this finding. Table 10a reports the reduction (S-C) in the median MCC value when source code metrics (C) alone are used, rather than source code metrics augmented by static mutation metrics $(\mathrm{S})$. Where this difference is negative, it means that static mutation metrics alone reduce the performance of the predictive model, and where it is positive, static mutation metrics alone improve performance. Similarly for Table 10b, except that this table concerns dynamic mutation metrics (D) alone. As can be seen, the beneficial effect of mutation metrics, in general, is lost when only static or only dynamic mutation metrics are used. This suggests that both static and dynamic mutation metrics are needed, and that their benefit comes from an interaction between the two. Static 
(a) Static

\begin{tabular}{l|c|c|c}
\hline & Apache & Eclipse & TelCom \\
\hline \multirow{2}{*}{ Naïve Bayes } & $\begin{array}{l}0.008 \\
(-0.028)\end{array}$ & $\begin{array}{l}0.450 \\
(0.003)\end{array}$ & $\begin{array}{l}<0.001 \\
(0.027)\end{array}$ \\
\hline \multirow{2}{*}{ Logistic Regression } & $\begin{array}{l}<0.001 \\
(-0.055)\end{array}$ & $\begin{array}{l}<0.001 \\
(-0.010)\end{array}$ & $\begin{array}{l}0.259 \\
(-0.009)\end{array}$ \\
\hline \multirow{2}{*}{ Random Forest } & $\begin{array}{l}0.036 \\
(0.025)\end{array}$ & $\begin{array}{l}0.010 \\
(0.012)\end{array}$ & $\begin{array}{l}0.427 \\
(0.013)\end{array}$ \\
\hline \multirow{2}{*}{$\mathrm{J} 48$} & $<0.001$ & $\begin{array}{l}0.030 \\
(-0.009)\end{array}$ & $\begin{array}{l}0.044 \\
(0.044)\end{array}$ \\
\hline
\end{tabular}

(b) Dynamic

\begin{tabular}{l|c|c|c}
\hline & Apache & Eclipse & TelCom \\
\hline \multirow{2}{*}{ Naïve Bayes } & $\begin{array}{l}0.569 \\
(-0.014)\end{array}$ & $\begin{array}{l}<0.001 \\
(-0.033)\end{array}$ & $\begin{array}{l}<0.001 \\
(0.039)\end{array}$ \\
\hline \multirow{2}{*}{ Logistic Regression } & $\begin{array}{l}0.555 \\
(0.017)\end{array}$ & $\begin{array}{l}<0.001 \\
(-0.019)\end{array}$ & $\begin{array}{l}0.368 \\
(-0.009)\end{array}$ \\
\hline \multirow{2}{*}{ Random Forest } & $\begin{array}{l}0.036 \\
(-0.052)\end{array}$ & $\begin{array}{l}0.101 \\
(0.012)\end{array}$ & $\begin{array}{l}0.048 \\
(0.036)\end{array}$ \\
\hline \multirow{2}{*}{$\mathrm{J} 48$} & $<0.001$ & $\begin{array}{l}0.429 \\
(-0.005)\end{array}$ & $\begin{array}{l}0.012 \\
(0.050)\end{array}$ \\
\hline
\end{tabular}

Table 10: RQ3. Two-tailed Wilcoxon test results are $p$ values that show the probability that we would obtain the difference in medians we observe, if (a) static or (b) dynamic mutation metrics were to have no significant effect on predictive performance (measured using MCC). The number in brackets is the difference between the median values; where positive it indicates static mutation metrics can improve predictive performance.

mutation metrics measure the number of simulated faults that a program may contain, while dynamic mutation metrics reflect the ability of the existing test suite to detect these simulated faults. In order to improve the performance of a predictive model, it seems intuitive that information will be needed on both the kind of faults the program may contain, and the ability of the test suite to detect them. The results for RQ3 support this observation; while static and dynamic mutation metrics alone are unhelpful, they are highly beneficial when combined.

\section{THREATS TO VALIDITY}

Our empirical study can be biased by three types of validity threats: construct validity, related to the agreement between a theoretical concept and a specific measuring device or procedure; conclusion validity, related to the ability to draw statistically correct conclusions; external validity, related to the ability to generalise the achieved results.

In order to satisfy construct validity a study has "to establish correct operational measures for the concepts being studied" [39]. Thus, the choice of the measures and how to collect them are crucial. We mitigated such a threat by using fault data that has been carefully collected (see Section 4.2) and used in previous empirical studies [23]. The source code metrics have been collected using JHawk [74], a robust tool for collecting these kind of metrics in Java systems [46]. To collect the mutation information we used the PITtest [14] tool since we want to perform our analysis on large subjects and it is the most scalable mutation testing tool. Since the results indicate that mutation can be helpful, they provide a lower bound on the performance of mutation testing, but further experimentation with different sets of mutation operators would be advisable; the lower bound can only be raised, because any mutation operators found to be universally unhelpful can simply be discarded for approaches to mutation-aware fault prediction.

In relation to conclusion validity, we carefully calculated the employed performance measures and applied statistical tests, verifying all the required assumptions.

The external validity of our study can be biased by the subjects we considered. Despite using both open source and industrial projects, we cannot claim that the conclusions reported apply to other software. The only way to mitigate this threat is to replicate the present study on other datasets. Moreover our analysis was performed on data belonging to the same software version, thus it is possible that these results might be valid only for the current version. To mitigate this threat we plan to investigate in our future work mutation based metrics both for next-releases [25] and cross-project fault predictions [48, 63].

\section{RELATED WORK}

Fault prediction is an active research field within Software Engineering. In 1971 Akiyama [2] proposed the earliest fault predictive model based on Lines of Code (LOC) for a software system developed at Fujitsu, estimating that a 1KLOC module was expected to contain approximatively 23 faults. Since then many predictive models and metrics have been investigated. Some recent literature reviews are available on both fault prediction models and metrics $[4,22,66]$. For the sake of space in the following we focus on work that investigated and compared the use of different sets of metrics as independent variables to build predictive models.

Besides LOC, other source code metrics (e.g., CK metrics [13]) and complexity metrics (e.g., Halsted metrics [24], McCabe's cyclomatic complexity [47], entropy metrics [28]) have been widely used and yield reasonable fault prediction performance $[36,49]$. All these metrics are based on software code, however other metrics can be obtained from different software artefacts. Ohlsson and Alberg reported on an empirical study carried out at Ericsson where metrics derived automatically from design documents were used to predict fault-prone modules [60]. Process metrics (e.g., number of commits, number of files changed, lines added/deleted) can be collected from versioning systems and have been shown to be effective in improving the accuracy of predictive models (see e.g., [31, 37, 52, 54, 58, 67]). Nagappan et al. [55] proposed a metric scheme to quantify organisational complexity, in relation to the product development process and investigated the merits of these metrics in building fault prediction models. The investigation carried out on data collected from Windows Vista revealed that organisational metrics were statistically significant predictors of failure-proneness, and the precision and recall measures for identifying failureprone binaries, using the organisational metrics, was significantly higher than using traditional metrics like churn, complexity, coverage, dependencies, and pre-release bug measures. Caglayan et al. [10] recently replicated the study of Nagappan et al. [55] on different large scale enterprise software. The results revealed that models based on organizational metrics performed better than models based on churn metrics but were outperformed by pre-release metric models. Thus, they concluded that the performance of different metric sets in building fault prediction models depends on the project's characteristics and the targeted prediction level. Recently the use of metrics based on technical dependen- 
cies among software modules and social information about developers' behaviour and interactions has also been investigated since these aspects may affect software quality $[6,7$, $78,64,79,41]$. Static test code metrics can be also captured by computing the same set of static code metrics that have been previously described from the source code of test cases. Nagappan [53] introduced a test metric suite called Software Testing and Reliability Early Warning metric suite (STREW) for estimating software quality. Further studies conducted by Nagappan et al. $[56,57]$ indicated that the STREW for Java metric suite can effectively predict software quality. Successively, Liljeson and Mohlin [43] assessed the effectiveness of the STREW suite in predicting faults contained in a large industrial software project developed by Ericsson. Their results showed that the combination of source code metrics and test metrics do not outperform using only source code metrics.

To the best of our knowledge only one study has been carried out to relate test executions during development with fault likelihood [30]. In his work, Herzig investigated whether metrics based on test failure bursts can be used to build pre- and post-release faults prediction models [30]. The results showed that test metrics collected during Windows 8 development outperform pre-release fault counts when predicting post-release faults. There has been previous work that used mutation testing in fault localisation [51][75] and various metrics (including test-based metrics) have been used to predict mutation scores [33]. These studies are the closest related previous work to the mutation-aware fault prediction approach introduced in the present paper. However, despite much work on different proposed metrics for fault prediction, the present paper is the first to use mutation metrics for fault prediction and to use fault prediction information related to the testing phase (i.e., mutation coverage and scores). The positive results we have found for this mutation-aware approach to predictive modelling are encouraging (both for related and for future work), suggesting that other such metrics may profitably be explored and could further improve the performance of fault prediction.

\section{CONCLUSIONS, IMPLICATIONS \& FU- TURE WORK}

We find that mutation-aware fault prediction can outperform traditional fault prediction, for a range of predictive modelling machine learning algorithms. Naturally, the effects vary, from system to system and from algorithm to algorithm, with different subsets of mutants contributing to the overall improvement. Nevertheless, in all cases, mutation metrics feature highly in the top 10 most influential metrics across all systems and algorithms, providing consistent evidence that mutation awareness is beneficial to predictive modelling performance. Our results also indicate that the best performance is obtained using a combination of both static and dynamic mutation metrics.

Naturally, we believe the primary implication of our findings lies in the potential benefit for fault prediction. However, we also believe that mutation-aware fault prediction could be valuable for mutation testing too: one critical problem for mutation testing lies in the selecting of a suitable set of mutation operators $[8,27,59]$. Since mutation awareness benefits fault prediction, the most desirable mutation operator set, $m$, for a given system under test, $s$, would include those that tend to improve fault prediction for $s$. That is, the set $m$ will be the most closely coupled to (detectable) real faults in $s$, by virtue of $m$ 's ability to predict the subsequent detection of real faults in $s$. This could allow us to tailor mutation operator selection to the likely detectable faults in the program under test. In this way our findings may have actionable implications for both mutation testing and for fault prediction. We hope that mutation-aware fault prediction will stimulate the creation of a symbiotic relationship between the two fields of study.

Finally, since we find that different systems benefit from different subsets of mutation metrics (and, indeed, different sets of source code metrics more generally), and that test awareness is important for best results, future work might consider other sets of mutants, mutation-aware metrics, and traditional metrics. Future work may also investigate other predictive modelling approaches and software systems to widen the generalisability of the results presented here.

\section{Acknowledgement}

This work was partly funded by the UK's Engineering and Physical Sciences Research Council (EPSRC) under grant numbers EP/L011751/1 and EP/J017515.

\section{REFERENCES}

[1] W. Afzal, R. Torkar, R. Feldt, and T. Gorschek. Prediction of faults-slip-through in large software projects: an empirical evaluation. Softw. Quality J., $22(1): 51-86,2014$.

[2] F. Akiyama. An example of software system debugging. In Proc. of the IFIP Congress, p 353-359, 1971.

[3] J. Andrews, L. Briand, and Y. Labiche. Is mutation an appropriate tool for testing experiments? In 27th Int'l Conf. on Softw. Eng., p 402-411, 2005.

[4] E. Arisholm, L. C. Briand, and E. B. Johannessen. A systematic and comprehensive investigation of methods to build and evaluate fault prediction models. J. Syst. Softw., 83(1):2-17, 2010.

[5] Y. Bejamini and Y. Hochberg. Controlling the false discovery rate: A practical and powerful approach to multiple testing. J. of the Royal statistical Soc. (Series B), 57(1):289-300, 1995.

[6] N. Bettenburg and A. E. Hassan. Studying the impact of social interactions on software quality. Empirical Softw. Engg., 18(2):375-431, 2013.

[7] C. Bird, N. Nagappan, P. Devanbu, H. Gall, and B. Murphy. Putting it all together: Using socio-technical networks to predict failures. In Int'l Symp. on Softw. Reliability Eng.. 2009.

[8] L. Bottaci and E. S. Mresa. Efficiency of mutation operators and selective mutation strategies: An empirical study. STVR, 9(4):205-232, Dec. 1999.

[9] L. Breiman. Random forests. Machine Learning, 45:5-32, 2001

[10] B. Caglayan, B. Turhan, A. Bener, M. Habayeb, A. Miransky, and E. Cialini. Merits of organizational metrics in defect prediction: An industrial replication. In IEEE Int'l Conf. on Softw. Eng., 2, 89-98, 2015.

[11] G. Canfora, A. D. Lucia, M. D. Penta, R. Oliveto, A. Panichella, and S. Panichella. Defect prediction as 
a multiobjective optimization problem. Softw. Test., Verif. Reliab., 25(4):426-459, 2015.

[12] C. Catal and B. Diri. A systematic review of software fault prediction studies. Expert systems with applications, 36(4):7346-7354, 2009.

[13] S. Chidamber and C. Kemerer. A metrics suite for object oriented design. IEEE Trans. on Softw. Eng., p 476-493, 1994.

[14] H. Coles. http://pitest.org/, 2015.

[15] S. Counsell and E. Nasseri. Java method calls in the hierarchy-uncovering yet another inheritance foible. CIT. J. of Computing and Information Technology, 18(2):159-165, 2010.

[16] D. R. Cox. The regression analysis of binary sequences. J. of the Royal Statistical Soc. Series B (Methodological), p 215-242, 1958.

[17] M. Dash and H. Liu. Feature selection for classification. Intelligent Data Analysis, 1(1-4):131 156, 1997.

[18] R. A. DeMillo, R. J. Lipton, and F. G. Sayward. Hints on test data selection: Help for the practical programmer. IEEE Computer, 11:31-41, 1978.

[19] N. E. Fenton and M. Neil. A critique of software defect prediction models. Softw. Eng., IEEE Trans. on, 25(5):675-689, 1999.

[20] G. Fraser and A. Zeller. Mutation-driven generation of unit tests and oracles. In Int'l Symp. on Softw. Testing and Analysis, p 147-158, Trento, Italy, 2010. ACM.

[21] M. Hall, E. Frank, G. Holmes, B. Pfahringer, P. Reutemann, and I. H. Witten. The weka data mining software: An update. In SIGKDD Explorations, 11(1), 2009.

[22] T. Hall, S. Beecham, D. Bowes, D. Gray, and S. Counsell. A systematic literature review on fault prediction performance in software engineering. IEEE Trans. Softw. Eng., 38(6):1276-1304, 2012.

[23] T. Hall, M. Zhang, D. Bowes, and Y. Sun. Some code smells have a significant but small effect on faults. ACM Trans. on Softw. Eng. and Methodology, 23(4):33, 2014.

[24] M. H. Halstead. Elements of Softw. Science (Operating and programming systems series). Elsevier Science Inc., NY, USA, 1977.

[25] M. Harman, S. S. Islam, Y. Jia, L. L. Minku, F. Sarro, and K. Srivisut. Less is more: Temporal fault predictive performance over multiple hadoop releases. In Search-Based Softw. Eng. - 6th Int'l Symp., p 240-246, 2014.

[26] M. Harman, Y. Jia, and W. B. Langdon. Strong higher order mutation-based test data generation. In $8^{\text {th }}$ European Softw. Eng. Conf. and the ACM SIGSOFT Symp. on the Foundations of Softw. Eng., p 212-222, 2011.

[27] M. Harman, Y. Jia, P. R. Mateo, and M. Polo. Angels and monsters: an empirical investigation of potential test effectiveness and efficiency improvement from strongly subsuming higher order mutation. In ACM/IEEE Int'l Conf. on Automated Softw. Eng., $\mathrm{p}$ 397-408. ACM, 2014.

[28] A. E. Hassan. Predicting faults using the complexity of code changes. In Proc. of the 31st Int'l Conf. on Softw. Eng., p 78-88, 2009.
[29] S. Henry and D. Kafura. Softw. structure metrics based on information flow. Softw. Eng., IEEE Trans. on, (5):510-518, 1981.

[30] K. Herzig. Using pre-release test failures to build early post-release defect prediction models. In Int'l Symp. on Softw. Reliability Eng., 300-311. IEEE, 2014.

[31] K. Herzig, S. Just, A. Rau, and A. Zeller. Predicting defects using change genealogies. In IEEE Int'l Symp. on Softw. Reliability Eng., 118-127, 2013.

[32] W. E. Howden. Weak mutation testing and completeness of test sets. Trans. on Softw. Eng., 8:371-379, 1982.

[33] K. Jalbert and J. Bradbury. Predicting mutation score using source code and test suite metrics. In Realizing Artificial Intelligence Synergies in Software Engineering (RAISE), 2012 First International Workshop on, pages 42-46, 2012.

[34] Y. Jia and M. Harman. An analysis and survey of the development of mutation testing. IEEE Trans. on Softw. Eng., 37(5):649 - 678, 2011.

[35] R. Just, D. Jalali, L. Inozemtseva, M. D. Ernst, R. Holmes, and G. Fraser. Are mutants a valid substitute for real faults in software testing? In Proc. of the SIGSOFT Int'l Symp. on Foundations of Softw. Eng. ACM, 2014.

[36] S. Kim. Defect, defect, defect: Defect prediction 2.0. In 8th Int'l Conf. on Predictive Models in Softw. Eng., p 1-2, 2012.

[37] S. Kim, T. Zimmermann, E. J. W. Jr., and A. Zeller. Predicting faults from cached history. In Int'l Conf. on Softw. Eng. , 489-498, 2007.

[38] K. N. King and A. J. Offutt. A FORTRAN language system for mutation-based software testing. Software Practice and Experience, 21:686-718, 1991.

[39] B. Kitchenham, L. Pickard, and S. L. Pfleeger. Case studies for method and tool evaluation. IEEE Softw., 12(4):52-62, 1995.

[40] R. Kohavi and G. H. John. Wrappers for feature subset selection. Artificial Intelligence, 97(1-2):273-324, 1997. Special issue on relevance.

[41] T. Lee, J. Nam, D. Han, S. Kim, and H. P. In. Micro interaction metrics for defect prediction. In $A C M$ SIGSOFT Symp. and the European Conf. on Foundations of Softw. Eng., 311-321, 2011.

[42] W. Li and S. Henry. Maintenance metrics for the object oriented paradigm. In Softw. Metrics Symp., Proc.., First Int'l, p 52-60. IEEE, 1993.

[43] M. Liljeson and A. Mohlin. Softw. defect prediction using machine learning on test and source code metrics. Thesis no: MECS-2014-06, 2006.

[44] M. Lorenz and J. Kidd. Object-oriented Softw. Metrics: A Practical Guide. Prentice-Hall, USA, 1994

[45] S. D. Martino, F. Ferrucci, C. Gravino, and F. Sarro. A genetic algorithm to configure support vector machines for predicting fault-prone components. In Product-Focused Softw. Process Improvement - 12th Int'l Conf., p 247-261, 2011.

[46] G. Mauša, T. Galinac Grbac, and D. Dalbelo Bašić. Data collection for software defect prediction - an exploratory case study of open source software projects. In Proc. of MIPRO CTI, p 513-519, 2015. 
[47] T. McCabe. A complexity measure. Softw. Eng., IEEE Trans. on, SE-2(4):308 - 320, dec. 1976.

[48] T. Menzies, A. Butcher, D. R. Cok, A. Marcus, L. Layman, F. Shull, B. Turhan, and T. Zimmermann. Local versus global lessons for defect prediction and effort estimation. IEEE Trans. Softw. Eng., 39(6):822-834, 2013.

[49] T. Menzies, Z. Milton, B. Turhan, B. Cukic, Y. Jiang, and A. B. Bener. Defect prediction from static code features: current results, limitations, new approaches. Autom. Softw. Eng., 17(4):375-407, 2010.

[50] Y. Jia and M. Harman. Milu: A customizable, runtime-optimized higher order mutation testing tool for the full $\mathrm{C}$ language. In Testing Academia and Industry Conf. - Practice and Research Techniques, 94-98, 2008.

[51] S. Moon, Y. Kim, M. Kim, and S. Yoo. Ask the mutants: Mutating faulty programs for fault localization. In IEEE Int'l Conf. on Softw. Testing, Verification and Validation, 153-162, 2014.

[52] R. Moser, W. Pedrycz, and G. Succi. A comparative analysis of the efficiency of change metrics and static code attributes for defect prediction. In Proc. Int'l Conf. on Softw. Eng., 181-190, 2008.

[53] N. Nagappan. A software testing and reliability early warning (STREW) metric suite. PhD thesis, 2005.

[54] N. Nagappan and T. Ball. Use of relative code churn measures to predict system defect density. In Proc. Int'l Conf. on Softw. Eng., 284-292, 2005.

[55] N. Nagappan, B. Murphy, and V. Basili. The influence of organizational structure on software quality: An empirical case study. In Proc. of the 30th Int'l Conf. on Softw. Eng., p 521-530, 2008.

[56] N. Nagappan, L. Williams, M. Vouk, and J. Osborne. Early estimation of software quality using in-process testing metrics: A controlled case study. SIGSOFT Softw. Eng. Notes, 30(4):1-7, May 2005.

[57] N. Nagappan, L. Williams, M. Vouk, and J. Osborne. Using in-process testing metrics to estimate post-release field quality. In Softw. Reliability. IEEE Int'l Symp. on, p 209-214, 2007.

[58] N. Nagappan, A. Zeller, T. Zimmermann, K. Herzig, and B. Murphy. Change bursts as defect predictors. In Softw. Reliability Eng., IEEE 21st Int'l Symp. on, $\mathrm{p}$ 309-318, 2010.

[59] A. J. Offutt, G. Rothermel, and C. Zapf. An experimental evaluation of selective mutation. In Int'l Conf. on Softw. Eng., p 100-107. IEEE, 1993.

[60] N. Ohlsson and H. Alberg. Predicting fault-prone software modules in telephone switches. IEEE Trans. Softw. Eng., 22(12):886-894, Dec. 1996.

[61] P. Oman, J. Hagemeister, and A. Ash, D. Definition and taxonomy for software maintainability. Technical Report 91-08-TR, University of Idaho, 1991.

[62] M. Papadakis and N. Malevris. Automatic mutation test case generation via dynamic symbolic execution. In Int'l Symp. on Softw. Reliability Eng., 2010.

[63] F. Peters, T. Menzies, and A. Marcus. Better cross company defect prediction. In Proc. of the 10th Working Conf. on Mining Softw. Repositories, $\mathrm{p}$ 409-418, 2013.

[64] M. Pinzger, N. Nagappan, and B. Murphy. Can developer-module networks predict failures? In Proc. ACM SIGSOFT Int'l Symp. on Foundations of Softw. Eng., 2-12, 2008.

[65] J. Quinlan. C4. 5: programs for machine learning, volume 1. Morgan kaufmann, 1993.

[66] D. Radjenović, M. Heričko, R. Torkar, and A. Živkovič. Softw. fault prediction metrics: A systematic literature review. Information and Softw. Technology, 55(8):1397-1418, 2013.

[67] F. Rahman and P. T. Devanbu. How, and why, process metrics are better. In 35th Int'l Conf. on Softw. Eng., p 432-441, 2013.

[68] F. Sarro, S. D. Martino, F. Ferrucci, and C. Gravino. A further analysis on the use of genetic algorithm to configure support vector machines for inter-release fault prediction. In Proc. of the ACM Symp. on Applied Computing,, p 1215-1220, 2012.

[69] B. H. Sellers. Object-Oriented Metrics. Measures of Complexity. Prentice Hall, 1996.

[70] M. Shepperd, D. Bowes, and T. Hall. Researcher bias: The use of machine learning in software defect prediction. Softw. Eng., IEEE Trans. on, 40(6):603-616, 2014.

[71] J. Śliwerski, T. Zimmermann, and A. Zeller. When do changes induce fixes? ACM sigsoft software engineering notes, 30(4):1-5, 2005.

[72] Q. Song, Z. Jia, M. Shepperd, S. Ying, and J. Liu. A general software defect-proneness prediction framework. Softw. Eng., IEEE Trans. on, 37(3):356-370, May 2011.

[73] M. Staats, G. Gay, and M. P. E. Heimdahl. Automated oracle creation support, or: How I learned to stop worrying about fault propagation and love mutation testing. In Int'l Conf. on Softw. Eng., 870-880, 2012.

[74] Virtualmachinery. JHawk the Java metrics tool, 2015.

[75] J. M. Voas. Pie: a dynamic failure-based technique. IEEE Trans. on Softw. Eng., 18(8):717-727, 1992.

[76] I. H. Witten and E. Frank. Data Mining: Practical machine learning tools and techniques. Morgan Kaufmann, 2005.

[77] M. R. Woodward and K. Halewood. From weak to strong, dead or alive? an analysis of some mutation testing issues. In $2^{\text {nd }}$ Workshop on Softw. Testing, Verification, and Analysis, 1988.

[78] T. Zimmermann and N. Nagappan. Predicting subsystem failures using dependency graph complexities. In Proc. of the The 18th IEEE Int'l Symp. on Softw. Reliability, p 227-236, 2007.

[79] T. Zimmermann and N. Nagappan. Predicting defects using network analysis on dependency graphs. In Proc. of the Int'l Conf. on Softw. Eng., 531-540, 2008.

[80] T. Zimmermann and N. Nagappan. Predicting defects with program dependencies. In Empirical Softw. Eng. and Measurement, Int'l Symp. on, 435-438, 2009. 